\title{
Does new-onset postoperative atrial fibrillation after coronary artery bypass grafting affect postoperative quality of life?
}

\author{
Sander Bramer, MD, ${ }^{\mathrm{a}} \mathrm{F}$. Joost ter Woorst, MD, ${ }^{\mathrm{a}}$ Martijn W. A. van Geldorp, MD, ${ }^{\mathrm{a}}$ \\ Krista C. van den Broek, MD, PhD, ${ }^{\text {b,c }}$ Jos G. Maessen, MD, PhD, ${ }^{\text {d }}$ Eric Berreklouw, MD, PhD, and \\ Albert H. van Straten, $\mathrm{MD}, \mathrm{PhD}^{\mathrm{a}}$
}

\begin{abstract}
Objectives: New-onset postoperative atrial fibrillation (POAF) is a common complication after cardiac surgery. We investigated the effect of POAF on quality of life after coronary artery bypass grafting.

Methods: All patients who underwent nonemergency coronary artery bypass grafting between March 2009 and January 2011 were requested to complete a Short Form-36 Health Survey before and 6 months after the procedure. Norm-based scores of 8 health status domains and 2 component summary scores were calculated. Only patients undergoing first-time coronary artery bypass grafting, with no history of atrial fibrillation, were included in the analyses.

\begin{abstract}
Results: Of 1608 patients, 360 (22.4\%) had POAF diagnosed. Twenty-eight patients died within half a year after the procedure $(1.7 \%$ in the no POAF group and $1.8 \%$ in the POAF group; $P=.90)$. After excluding these patients, data from 1580 patients were analyzed. Preoperative questionnaires were returned by $66 \%$ of the patients and postoperative questionnaires by $65 \%$. Preoperative scores did not differ between patients with and without POAF in any subcategory (0/10). After the procedure, 4 of 10 scores were worse in the group with POAF compared to patients without POAF. Patients without POAF improved in all subcategories (10/10) after the procedure, whereas those with POAF did so in only 7 of 10 . Multilinear regression showed POAF to be an independent negative predictor for improved quality of life 6 months postoperatively in 7 of 10 subcategories, including both mental and physical component summary scores.
\end{abstract}

Conclusions: New-onset POAF does affect 6-month postoperative mental and physical health, possibly warranting more aggressive POAF treatment. (J Thorac Cardiovasc Surg 2013;146:114-8)

It is well known that new-onset postoperative atrial fibrillation (POAF) after coronary artery bypass grafting (CABG) is related to morbidity and mortality. ${ }^{1-5}$ Although the negative effects of atrial fibrillation on quality of life (QoL) have been demonstrated in the general population, ${ }^{6}$ no data are available concerning new-onset atrial fibrillation after cardiac surgery. There have been several reports on QoL after cardiac surgery. Among those, few reported both preoperative and postoperative data, and none reported the effects of POAF on QoL. ${ }^{7}$ The aim of this study was therefore to investigate the independent effect of POAF on health-related QoL after CABG by comparing scores

\footnotetext{
From the Departments of Cardio-Thoracic Surgery ${ }^{\mathrm{a}}$ and Education and Research, ${ }^{\mathrm{b}}$ Catharina Hospital, Eindhoven, The Netherlands; the Department of Medical Psychology, ${ }^{\mathrm{c}}$ Center of Research on Psychology in Somatic Diseases, Tilburg University, Tilburg, The Netherlands; and the Department of Cardiothoracic Surgery, ${ }^{\mathrm{d}}$ Cardiovascular Research Institute Maastricht (CARIM), Maastricht University Medical Center (MUMC), Maastricht, The Netherlands.

Disclosures: Authors have nothing to disclose with regard to commercial support.

Received for publication April 4, 2012; revisions received May 14, 2012; accepted for publication June 15, 2012; available ahead of print July 27, 2012.

Address for reprints: Sander Bramer, MD, Department of Cardiothoracic Surgery, Catharina Hospital, Michelangelolaan 2, Postbus 1350, 5602 ZA Eindhoven, The Netherlands (E-mail: sander.bramer@cze.nl).

0022-5223/\$36.00

Copyright (C) 2013 by The American Association for Thoracic Surgery http://dx.doi.org/10.1016/j.jtcvs.2012.06.045
}

from preoperative and postoperative responses to the Short Form-36 Health Survey version 2.0 (Dutch, SF-36v2) in patients with and without POAF.

\section{MATERIALS AND METHODS Patients}

The institutional research review board approved this study and waived the need for patient consent. This study included patients who consecutively underwent nonemergency nonreoperative CABG in a single center in the Netherlands (Catharina Hospital, Eindhoven) between March 2009 and January 2011. Only patients with documented preoperative sinus rhythm and without a history of atrial fibrillation were included. Demographic data and risk factors for postoperative morbidity and mortality, including POAF, were prospectively collected in a computerized database. POAF was defined as postoperative occurrence of atrial fibrillation for at least 30 minutes. Mortality data were gathered from databases of health insurance companies and by information requests to general practitioners or municipal authorities.

\section{SF-36v2 Questionnaire}

In January 2009, we started a quality control program in our department. As a part of this program, all patients undergoing nonemergency cardiac surgery are requested to complete the SF-36v2 before the operation and again at 6 and 12 months after the operation. This survey is a widely used, reliable, and validated standardized questionnaire to measure both physical and mental status of a patient. ${ }^{8,9}$ The SF-36v2 assesses 8 health status domains: Physical Functioning (ability to perform all types of physical activities without limitations from health), Role Physical (problems with daily activities or work as a result of physical health), Bodily Pain 


\section{Abbreviations and Acronyms \\ $\mathrm{CABG}=$ coronary artery bypass grafting \\ $\mathrm{POAF}=$ postoperative atrial fibrillation \\ $\mathrm{QoL}=$ quality of life \\ SF- $\quad=$ Short Form-36 Health Survey version 2 \\ 36v2 (Dutch)}

(pain or limitations imposed by pain), General Health (perception of general health), Vitality, Social Functioning, Role Emotional (problems with work or daily activities as a result of emotional problems), and Mental Health (feelings of nervousness or depression). From these 8 subscales, the Physical and Mental Component Summary scores can be generated, reflecting the patient's overall physical and mental health status. ${ }^{8}$ Missing data were estimated and substituted according to the half-scale rule. ${ }^{8}$ Subcategory and component scores were transformed to a scale from 0 to 100 , with higher scores indicating a better health status. These transformed scores were then standardized to the general Dutch population $($ mean $=50,1$ standard deviation $=10)$.

\section{Operative Technique and Postoperative Policy}

It was our policy to continue the use of $\beta$-blocking agents preoperatively until the day of the operation. All patients received short-acting anesthetic drugs to facilitate early extubation. Normothermic extracorporeal circulation was performed with nonpulsatile flow. Intermittent cold crystalloid cardioplegia (St Thomas solution) or intermittent warm blood cardioplegia was used to induce and maintain cardioplegic arrest, according to the surgeon's preference. Sixteen percent of all operations were performed without the use of extracorporeal circulation. After the operation, all patients without contraindication for $\beta$-blocking agents received metoprolol as prophylaxis against atrial fibrillation. Rhythm was continuously registered for at least 48 postoperative hours. Afterward, until discharge to the referring hospital or home, registration was performed at least 3 times a day, or continuously in case of dysrhythmia. POAF was treated by rate control with metoprolol in hemodynamically stable patients, and electrocardioversion or cardioversion with amiodarone was attempted otherwise. If atrial fibrillation persisted for longer than 48 hours, an anticoagulant was started. The referring cardiologist determined the atrial fibrillation treatment regimen after discharge, according to the guidelines of the American College of Cardiology, the American Heart Association, and the European Society of Cardiology. ${ }^{11}$

\section{Statistical Analyses}

Patient demographics and characteristics were compared with independent $t$ tests for continuous variables, and categoric variables were compared with the $\chi^{2}$ test. The dependent $t$ test was used to compare differences in preoperative and postoperative SF-36v2 scores within the POAF and non-POAF groups. SF-36v2 scores of patients with and without POAF were compared with the independent $t$ test. A multiple linear regression model was used to investigate the independent effect of POAF on each subscale of the SF-36v2 survey 6 months postoperatively, adjusted for the preoperative scores. In a previous article, we reported several independent risk factors for POAF, such as body mass index, age, and male sex. ${ }^{12}$ To account for possible confounders, these were included in the current regression model. All statistical analyses were done with the SPSS statistical software package (version 19.0; IBM Corporation, Armonk, NY).

\section{RESULTS}

After excluding emergency and reoperative procedures and patients with a history of atrial fibrillation $(n=60$;
$3.6 \%)$ or pacemaker $(\mathrm{n}=9 ; 0.5 \%), 1608$ patients were eligible for analysis. Of those 1608 patients, 360 (22.4\%) had POAF diagnosed. Twenty-eight patients died within half a year after the procedure $(1.7 \%$ in the non-POAF group vs $1.8 \%$ in the POAF group; $P=.90$ ). Patients with POAF were older than those without (69.3 vs 63.8 years; Table 1).

Compared with patients who did not complete a preoperative SF-36v2 form, patients who did were more frequently men $(79.0 \%$ vs $74.5 \% ; P=.040)$. Death rate within half a year did not differ between responders and nonresponders $(1.9 \%$ vs $1.5 \% ; P=.55)$. No differences in frequency of POAF $(22.7 \%$ vs $21.8 \% ; P=.70)$ and other demographic and other characteristics were observed between responders and nonresponders.

Preoperative scores of patients alive at 6 months after CABG were significantly better in 6 subcategories, compared with patients who did not survive the first half year. After excluding the patients who died within half a year after surgery, data of 1580 patients were used for further analyses.

Preoperative SF-36v2 forms were returned by 1043 patients $(66 \%)$. From 977 forms $(94 \%)$, all subcategories could be analyzed. Postoperative SF-36v2 forms were returned by 1019 patients (65\%), and from 978 forms (96\%), all subcategories could be analyzed. Both preoperative and 6-month postoperative forms were returned by 710 patients $(45 \%)$. The response percentages at both preoperative and 6-month postoperative intervals were equal for patients with and without $\operatorname{POAF}(P=.77$ and $P=.59$, respectively).

TABLE 1. Demographic and clinical characteristics

\begin{tabular}{lccc}
\hline \multicolumn{1}{c}{ Variable } & No POAF $(\mathbf{n}=\mathbf{1 2 4 8})$ & POAF $(\mathbf{n}=\mathbf{3 6 0})$ & $\boldsymbol{P}$ value \\
\hline Age $(\mathrm{y})$ & $63.8 \pm 9.9$ & $69.3 \pm 8.4$ & $<.001$ \\
Male & 78.2 & 75.0 & .20 \\
BMI $\left(\mathrm{kg} / \mathrm{m}^{2}\right)$ & $27.4 \pm 4.0$ & $27.6 \pm 4.0$ & .41 \\
Diabetes & 21.2 & 21.4 & .95 \\
Hypertension & 56.2 & 56.4 & .94 \\
COPD & 9.8 & 11.1 & .46 \\
PVD & 15.1 & 16.9 & .39 \\
Previous CVA & 4.2 & 3.9 & .77 \\
Normal LV & 84.1 & 80.0 & .064 \\
$\quad$ function & & & \\
CABG with ECC & 83.0 & 86.1 & .16 \\
Grafts (no.) & $3.46 \pm 1.0$ & $3.54 \pm 1.0$ & .18 \\
ECC (min)* & $69.0 \pm 23$ & $69.4 \pm 22$ & .77 \\
AoX (min)* & $48.2 \pm 23$ & $47.1 \pm 16$ & .42 \\
\hline
\end{tabular}

Categoric variables are presented as percentage; continuous variables are presented as mean \pm SD. Distribution of risk factors of postoperative atrial fibrillation and other possible risk factors for postoperative morbidity and mortality in patients with and without POAF. $A o X$, Aortic crossclamp time; $B M I$, body mass index; $C A B G$, coronary artery bypass grafting; $C O P D$, chronic obstructive pulmonary disease; $C V A$, cerebrovascular accident; $E C C$, extracorporeal circulation; $L V$, left ventricular; $P O A F$, postoperative atrial fibrillation; $P V D$, peripheral vascular disease. *Analyzed in patients with on-pump surgery $(\mathrm{n}=1351)$. 
TABLE 2. Preoperative and postoperative norm-based SF-36 scores in patients with or without postoperative atrial fibrillation

\begin{tabular}{|c|c|c|c|c|c|c|}
\hline & \multicolumn{3}{|c|}{ No POAF $(n=554)$} & \multicolumn{3}{|c|}{ POAF $(n=156)$} \\
\hline & Before & $6 \mathrm{mo}$ & $P$ value & Before & $6 \mathrm{mo}$ & $P$ value \\
\hline $\begin{array}{l}\text { Physical Component } \\
\text { Summary }\end{array}$ & 41.7 & 49.0 & $<.001$ & 42.2 & 47.6 & $<.001$ \\
\hline $\begin{array}{l}\text { Mental Component } \\
\text { Summary }\end{array}$ & 45.1 & 46.7 & $<.001$ & 45.9 & 45.8 & .60 \\
\hline Physical Functioning & 38.8 & 47.4 & $<.001$ & 38.4 & 44.1 & $<.001$ \\
\hline Role Physical & 41.8 & 46.3 & $<.001$ & 42.9 & 44.9 & .004 \\
\hline Bodily Pain & 44.0 & 51.1 & $<.001$ & 44.0 & 50.7 & $<.001$ \\
\hline General Health & 44.5 & 47.6 & $<.001$ & 45.3 & 46.9 & .010 \\
\hline Vitality & 43.7 & 48.3 & $<.001$ & 44.0 & 47.5 & $<.001$ \\
\hline Social Functioning & 43.2 & 48.6 & $<.001$ & 45.3 & 47.8 & .009 \\
\hline Role Emotional & 45.2 & 47.5 & $<.001$ & 45.6 & 45.8 & .80 \\
\hline Mental Health & 42.2 & 44.9 & $<.001$ & 42.3 & 43.6 & .11 \\
\hline
\end{tabular}

In patients without POAF, scores of all SF-36v2 subscales improved after 6 months, whereas in patients with POAF, Role Emotional, Mental Health, and Mental Component Summary scores did not improve (Table 2). For all components $(10 / 10)$ of the preoperative SF-36v2 survey, scores did not differ between patients with and without POAF (Figure 1). For 4 of 10 of the components of the 6-month postoperative SF-36v2 survey, scores were worse in patients with POAF than in those without POAF (Physical Component Summary, $P=.032$; Physical Functioning, $P=.003$; Role Physical, $P=.033$; and Role Emotional, $P=.012$; Figure 2). When only data of patients who completed both preoperative and postoperative surveys $(\mathrm{n}=710)$ were analyzed, Physical Component Summary $(P=.037)$, Physical Functioning $(P=.003)$, and Role Emotional $(P=.021)$ scored significantly worse. Multivariate linear regression showed POAF to be an independent negative predictor for improvement in 7 of 10 subcategories 6 months after the operation (Table 3).

\section{DISCUSSION}

In addition to prolonging survival, improvement of QoL is one of the major goals in cardiac surgery. Our results indicate that new-onset atrial fibrillation after CABG has a negative impact on QoL.

Preoperative overall physical health (Physical Component Summary score) did not differ between patients who developed POAF and those who did not. Physical Component Summary score improved in both groups, but the improvement was more pronounced in patients without POAF: after 6 months, physical health was significantly better in patients without POAF than in those with POAF. Patients with POAF had lower scores on the Physical Functioning and Role Physical subscales, which could account for this difference.

Preoperative overall mental health (Mental Component Summary score) did not differ between POAF and nonPOAF patient groups. Only patients without POAF, however, showed improved overall mental health after 6 months. Lack of improvement in the Role Emotional and Mental Health subscales seems to account for the failure to improve overall mental health among patients with POAF 6 months after CABG.

Patients who developed POAF were older, which independently predicts a worse postoperative QoL. ${ }^{13,14}$ By adjusting for age, and other predictors of POAF, however, we showed POAF to be an independent negative predictor of postoperative improvement in 7 of 10 subcategories, including both the Mental and Physical Component Summary scores. POAF thus was related to poor QoL independent of body mass index, age, and sex.

This study was not designed to determine why patients with POAF had a lower QoL. Palpitations, side effects of medical treatment, and complications of atrial fibrillation such as stroke or anticoagulant-related bleeding all could account for the impaired health of patients with POAF relative to those without POAF. Fear of complications may also play a role.

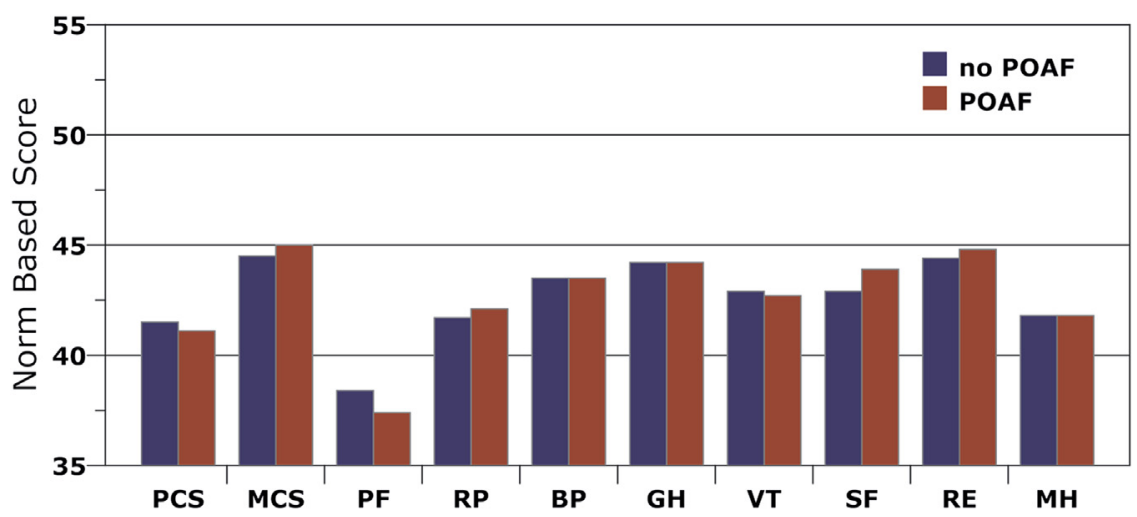

FIGURE 1. Preoperative Short Form-36 Health Survey version 2 (Dutch) norm based scores for patients without $(\mathrm{n}=807)$ and with (n $=236)$ postoperative atrial fibrillation, showing no significant difference. $B P$, Bodily Pain; $G H$, General Health; $M C S$, Mental Component Summary; $M H$, Mental Health; $P C S$, Physical Component Summary; $P F$, Physical Functioning; $R E$, Role Emotional; $R P$, Role physical; $S F$, Social Functioning; VT, Vitality. 


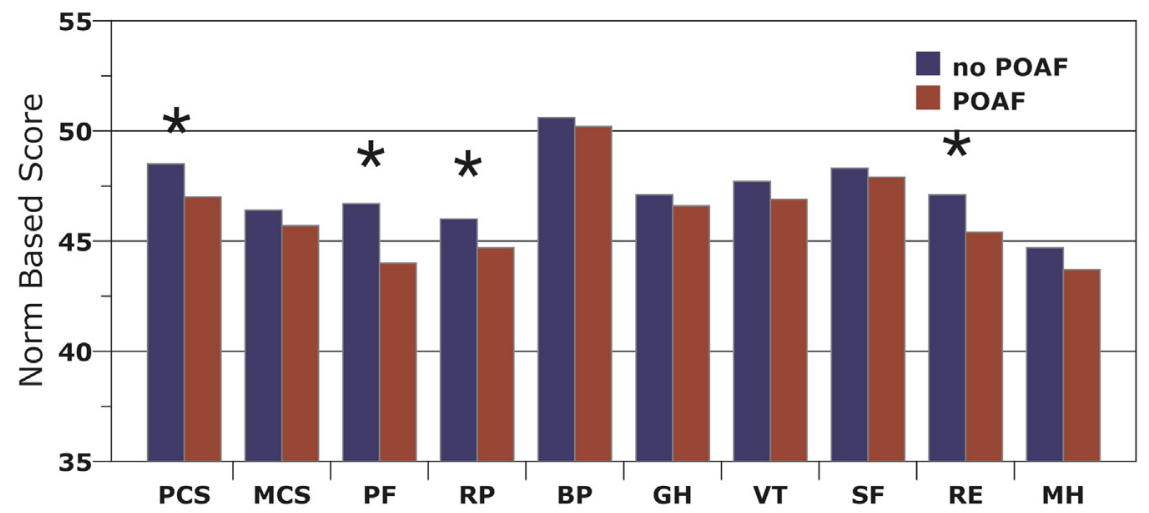

FIGURE 2. Six-month postoperative norm-based Short Form-36 Health Survey version 2 (Dutch) scores for patients without ( $\mathrm{n}=795$ ) and with postoperative atrial fibrillation $(\mathrm{n}=224)$, showing significant worse scores on Physical Component Summary, Physical Functioning, Role Physical, and Role Emotional components in patients with postoperative atrial fibrillation compared with those without. BP, Bodily Pain; GH, General Health; $M C S$, Mental Component Summary; $M H$, Mental Health; PCS, Physical Component Summary; PF, Physical Functioning; RE, Role Emotional; $R P$, Role Physical; $S F$, Social Functioning; VT, Vitality.

The extent of POAF most likely determines the extent of impairment in health outcome, although no previous study has investigated this issue. Unfortunately, in our database no data were available on the extent of POAF, and that is one of the limitations of this study. The impossibility of excluding patients with clinically irrelevant POAF (eg, only 1 episode of 30 minutes) warrants further research into the effect of duration of POAF on QoL. Furthermore, onset of POAF after discharge was not registered, and therefore its effect could not be analyzed.

Only $66 \%$ of the patients returned the preoperative SF-36v2 questionnaire. Similar studies have reported comparable percentages, although group numbers were smaller. ${ }^{15-18}$ Care should be taken in applying the results to the whole CABG group, because predictors of QoL could be different between responders and nonresponders. The response percentage was equal in

TABLE 3. Multiple linear regression analyses for prediction of improvement of 6-month postoperative Short Form-36 Health Survey version 2 (Dutch) scores by postoperative atrial fibrillation

\begin{tabular}{lcccc}
\hline & B & SE B & $\boldsymbol{\beta}$ & $\boldsymbol{P}$ value \\
\hline Physical Component Summary & -2.3 & 0.96 & -0.097 & .017 \\
Mental Component Summary & -2.2 & 0.90 & -0.099 & .014 \\
Physical Functioning & -3.1 & 1.06 & -0.115 & .004 \\
Role Physical & -3.0 & 0.84 & -0.140 & $<.001$ \\
Bodily Pain & -1.1 & 1.13 & -0.039 & .321 \\
General Health & -2.1 & 0.93 & -0.089 & .023 \\
Vitality & -1.7 & 1.06 & -0.062 & .12 \\
Social Functioning & -3.8 & 1.18 & -0.125 & .001 \\
Role Emotional & -2.7 & 0.92 & -0.117 & .003 \\
Mental Health & -1.6 & 0.87 & -0.072 & .070 \\
\hline
\end{tabular}

Results of multiple linear regression analyses for prediction of improvement of normbased scores of components of the 6-month postoperative SF-36 survey compared with the preoperative survey by postoperative atrial fibrillation. Postoperative atrial fibrillation and known risk factors for it (body mass index, age, sex) are included in the model.
POAF and non-POAF groups, however, and any potential difference in predictors of QoL by nonresponders would therefore be expected to be the same in the POAF and non-POAF groups.

Interestingly, from our analyses we found that preoperative health status predicts survival within 6 months after the procedure. The rationale behind this finding is not within the scope of this study but certainly deserves more profound research. Others have reported similar findings with less specific parameters of QoL, such as preoperative New York Heart Association class.

Rhythm surgery in patients with symptomatic atrial fibrillation improves $\mathrm{QoL}^{19-22}$; whether reduction of the occurrence of POAF or more aggressive treatment of POAF will improve postoperative QoL needs to be investigated in well-designed randomized clinical trials.

\section{CONCLUSIONS}

New-onset POAF is negatively correlated with mental and physical health 6 months after CABG, independent of age, sex, and body mass index.

\section{References}

1. Almassi GH, Schowalter T, Nicolosi AC, Aggarwal A, Moritz TE, Henderson WG, et al. Atrial fibrillation after cardiac surgery: a major morbid event? Ann Surg. 1997;226:501-11.

2. Bramer S, van Straten AH, Soliman Hamad MA, Berreklouw E, Martens EJ, Maessen JG. The impact of new-onset postoperative atrial fibrillation on mortality after coronary artery bypass grafting. Ann Thorac Surg. 2010;90: 443-9.

3. Mathew JP, Parks R, Savino JS, Friedman AS, Koch C, Mangano DT, et al. Atria fibrillation following coronary artery bypass graft surgery: predictors, outcomes, and resource utilization. MultiCenter Study of Perioperative Ischemia Research Group. JAMA. 1996;276:300-6.

4. Shen J, Lall S, Zheng V, Buckley P, Damiano RJ Jr, Schuessler RB. The persistent problem of new-onset postoperative atrial fibrillation: a singleinstitution experience of two decades. J Thorac Cardiovasc Surg. 2011;141: 559-70.

5. Almassi GH, Pecsi SA, Collins JF, Shroyer AL, Zenati MA, Grover FL. Predictors and impact of postoperative atrial fibrillation on patients' outcomes: 
a report from the Randomized On Versus Off Bypass trial. J Thorac Cardiovasc Surg. 2012;143:93-102.

6. Thrall G, Lane D, Carroll D, Lip GY. Quality of life in patients with atrial fibrillation: a systematic review. Am J Med. 2006;119:448.e1-19.

7. Noyez L, de Jager MJ, Markou AL. Quality of life after cardiac surgery: underresearched research. Interact Cardiovasc Thorac Surg. 2011;13:511-5.

8. Ware JE, Kosinski MA, Bjorner JB, Turner-Bowker DM, Gandek B, Maruish ME. User's manual for the SF-36v2 Health Survey 2nd ed. Lincoln (RI): Quality Metric; 2007.

9. Stansfeld SA, Roberts R, Foot SP. Assessing the validity of the SF-36 General Health Survey. Qual Life Res. 1997;6:217-24.

10. Aaronson NK, Muller M, Cohen PD, Essink-Bot ML, Fekkes M, Sanderman R, et al. Translation, validation, and norming of the Dutch language version of the SF-36 health survey in community and chronic disease populations. J Clin Epidemiol. 1998;51:1055-68.

11. Fuster V, Rydén LE, Asinger RW, Cannom DS, Crijns HJ, Frye RL, et al., ACC/ AHA/ESC guidelines for the management of patients with atrial fibrillation. A report of the American College of Cardiology/American Heart Association Task Force on Practice Guidelines and the European Society of Cardiology Committee for Practice Guidelines and Policy Conferences (Committee to develop guidelines for the management of patients with atrial fibrillation) developed in collaboration with the North American Society of Pacing and Electrophysiology. Eur Heart J. 2001;22:1852-923.

12. Bramer S, van Straten AH, Soliman Hamad MA, Berreklouw E, van den Broek KC, Maessen JG. Body mass index predicts new-onset atrial fibrillation after cardiac surgery. Eur J Cardiothorac Surg. 2011;40:1185-90.

13. Rumsfeld JS, Ho PM, Magid DJ, McCarthy M Jr, Shroyer AL, MaWhinney S, et al. Predictors of health-related quality of life after coronary artery bypass surgery. Ann Thorac Surg. 2004;77:1508-13.
14. Koch CG, Khandwala F, Cywinski JB, Ishwaran H, Estafanous FG, Loop FD, et al. Health-related quality of life after coronary artery bypass grafting: A gender analysis using the Duke Activity Status Index. J Thorac Cardiovasc Surg. 2004;128:284-95.

15. El Baz N, Middel B, van Dijk JP, Boonstra PW, Reijneveld SA. Coronary artery bypass graft $(\mathrm{CABG})$ surgery patients in a clinical pathway gained less in healthrelated quality of life as compared with patients who undergo CABG in a conventional-care plan. J Eval Clin Pract. 2009;15:498-505.

16. BØ Jensen, Hughes P, Rasmussen LS, Pedersen PU, Steinbrüchel DA. Health-related quality of life following off-pump versus on-pump coronary artery bypass grafting in elderly moderate to high-risk patients: a randomized trial. Eur J Cardiothorac Surg. 2006;30:294-9.

17. Lie I, Arnesen H, Sandvik L, Hamilton G, Bunch EH. Health-related quality of life after coronary artery bypass grafting. The impact of a randomised controlled home-based intervention program. Qual Life Res. 2009;18:201-7.

18. Grady KL, Lee R, Subačius H, Malaisrie SC, McGee EC Jr, Kruse J, et al. Improvements in health-related quality of life before and after isolated cardiac operations. Ann Thorac Surg. 2011;91:777-83.

19. Lundberg C, Albåge A, Carnlöf C, Kennebäck G. Long-term health-related quality of life after maze surgery for atrial fibrillation. Ann Thorac Surg. 2008;86:1878-82.

20. Jessurun ER, van Hemel NM, Defauw JA, Stofmeel MA, Kelder JC, de la Rivière $\mathrm{AB}$, et al. Results of maze surgery for lone paroxysmal atrial fibrillation. Circulation. 2000;101:1559-67.

21. Lönnerholm S, Blomström P, Nilsson L, Oxelbark S, Jideus L, BlomströmLundqvist C. Effects of the maze operation on health-related quality of life in patients with atrial fibrillation. Circulation. 2000;101:2607-11.

22. Bagge L, Blomstrom P, Nilsson L, Einarsson GM, Jidéus L, BlomströmLundqvist C. Epicardial off-pump pulmonary vein isolation and vagal denervation improve long-term outcome and quality of life in patients with atrial fibrillation. J Thorac Cardiovasc Surg. 2009;137:1265-71. 